\title{
Propuesta metodológica para la evaluación de la vulnerabilidad social en poblaciones afectadas por el peligro de inundación: el caso de Águilas (Murcia, sureste ibérico)
}

\author{
Alfredo Pérez Morales \\ Francisca Navarro Hervás \\ Yolanda Álvarez Rogel \\ Universidad de Murcia \\ alfredop@um.es \\ franaher@um.es \\ yalvarez@um.es
}

Recepción: julio de 2014

Aceptación: marzo de 2015

\section{Resumen}

En esta investigación se realiza una valoración de la vulnerabilidad social del riesgo de inundación por avenida mediante la construcción de un índice en una población del litoral murciano. Para ello se tuvieron en cuenta los principales indicadores socioeconómicos y sociodemográficos disponibles en el INE (Instituto Nacional de Estadística). Mediante la aplicación de un análisis multivariante sobre los indicadores elegidos, se redujo el número de variables y se hallaron los factores intervinientes ocultos. De la combinación de estos con el resultado de una encuesta de percepción previa, se obtuvo el valor final de vulnerabilidad social mejorando el número de dimensiones que se suelen considerar en la construcción de dichos índices. Con los resultados se elaboró una cartografía a nivel de sección censal, a fin de identificar aquellos sectores que, por su condición social evaluada mediante el índice, son susceptibles de experimentar un mayor impacto por inundación.

Palabras clave: vulnerabilidad; social; riesgo; inundación; evaluación.

Resum. Proposta metodològica per a l'avaluació de la vulnerabilitat social en poblacions afectades pel perill d'inundació: el cas d'Águilas (Múrcia, sud-est ibèric)

En aquesta recerca es duu a terme una valoració de la vulnerabilitat social del risc de patir una inundació per avinguda mitjançant la construcció d'un índex en una població del litoral murcià. Amb aquesta finalitat es van tenir en compte els indicadors socioeconòmics i sociodemogràfics principals disponibles per part de l'INE (Instituto Nacional de Estadística) els quals caracteritzen l'àmbit d'estudi. Mitjançant l'aplicació d'una anàlisi multivariant sobre els indicadors seleccionats es va reduir el nombre de variables i es van acotar els factors que hi intervenen de manera oculta. De la combinació d'aquests amb el resultat d'una enquesta de percepció realitzada prèviament, es va obtenir el valor final de la vulnerabilitat social, amb la 
qual cosa es va millorar el nombre de dimensions que se solen considerar en la construcció d'aquests índexs. En darrer terme, es va elaborar una cartografia per seccions censals a fi i efecte d'identificar aquells sectors que, atesa la seva condició social avaluada mitjançant l'índex en qüestió, són susceptibles d'experimentar un impacte major per episodis d'inundació.

Paraules clau: vulnerabilitat; social; risc; inundació; avaluació.

Résumé. Proposition méthodologique pour l'évaluation de la vulnérabilité sociale des populations affectées par des risques d'inondation: le cas d'Águilas (Murcie, sud-est ibérique)

Dans cette recherche, nous avons effectuée une évaluation de la vulnérabilité sociale du risque d'inondation par crue en construisant un indice dans un village de la côte de Murcie. A cet effet, nous avons tenu compte les principaux indicateurs démographiques et socio-économiques disponibles à partir de l'INE (Institut National de la Statistique) qui caractérisent la zone d'étude. En appliquant une analyse multivariante des indicateurs sélectionnés, nous réduit le nombre de variables et rendu manifeste l'intervention de facteurs occultes. La combinaison de ces derniers avec les résultats d'une enquête de perception menée précédemment nous a permis d'obtenir la valeur finale de la vulnérabilité sociale en améliorant le nombre de dimensions qui sont habituellement considérées dans la construction de ces indices. Enfin nous avons élaboré une cartographie au niveau des secteurs de recensement afin d'identifier les secteurs qui, compte tenu de leur statut social évalué à l'aide de l'indice en question, sont susceptibles d'éprouver un plus grand impact lors d'épisodes d'inondation.

Mots-clés: vulnérabilité; sociale; risque; inondation; évaluation.

Abstract. A methodological proposal for assessing social vulnerability in populations affected by the risk of flooding: The case of Aguilas, Murcia, in the southeast Iberian peninsula

This paper assesses social vulnerability associated with flooding in a coastal population of Murcia by building an index. The main socio-economic and demographic indicators are taken into account in the data sources. A factor analysis was performed on the selected indicators to infer and reduce the number of variables involved. To obtain the social vulnerability index, the indicators were combined with the results of a previously administered perception survey, thus improving the number of dimensions usually considered in the construction of these indices. Finally, social vulnerability was mapped on a census tract level to identify sectors that are likely to be affected by flooding and their degree of social vulnerability.

Keywords: vulnerability; social; risk; flooding; assessment.

\section{Sumario}

\section{Introducción}

2. Localización y características del área de estudio

3. Objetivo y metodología

4. Procedimiento para la construcción del índice de vulnerabilidad social y su representación cartográfica
5. Resultados y discusión

6. Conclusiones

Referencias bibliográficas

Apéndice 


\section{Introducción}

La evaluación y la cartografía de la vulnerabilidad social al riesgo con origen natural, constituye hoy una de las herramientas de mayor utilidad para la identificación y el análisis de los problemas relacionados con los desastres ambientales. Sin embargo, el proceso de consolidación experimentado hasta alcanzar dicha posición ha sido relativamente lento y ha motivado, en la mayoría de las ocasiones, un enfoque en las estrategias de mitigación que no termina por resolver de manera efectiva esas cuestiones.

En un primer momento, la evaluación del riesgo se centró en el estudio de la peligrosidad de los sucesos extremos, quedando relegado el papel de la exposición y la vulnerabilidad a un segundo término, tal y como señalaron diferentes autores (Cutter, 1996; Cardona, 2005; Adger, 2006; Calvo, 2001; Camarasa y López, 2008; Perles; 2010). No sorprende, por tanto, que durante buena parte del siglo pasado la mayoría de los desarrollos metodológicos apuntaran a la estimación de cuestiones relativas al factor físico del riesgo y, en consecuencia, las estrategias frente a este último se basaran en actuaciones de carácter estructural en la mayoría de los casos, tanto en el ámbito nacional como internacional.

Con motivo de la publicación de la Estrategia Internacional para la Reducción de los Riesgos Naturales, en la que se consensuó el concepto de vulnerabilidad y se incluyó como un componente principal en los estudios de riesgo, parece que el escenario anteriormente comentado empezó a cambiar (Adger, 2006; Birkmann, 2006; Cutter y Finch, 2008). Posteriormente, dicha modificación quedó reforzada con el Marco de Acción de Hyogo 2005-2015, donde «los gobiernos de todo el mundo se comprometen a tomar medidas para reducir la vulnerabilidad frente a las amenazas naturales» (EIRD, 2005).

El resultado de esa evolución se ha traducido en una auténtica explosión de trabajos que aplican nuevas metodologías encaminadas a la evaluación y a la cartografía de la vulnerabilidad a diversas amenazas de origen natural. Al nivel internacional se pueden mencionar: Estados Unidos (Clark et al., 1998; Cutter et al., 2003; Wu et al., 2002; Chakraborty et al., 2005; Olfert et al., 2006; Rygel et al., 2006; Kleinosky et al., 2007; Yarnal, 2007), Reino Unido (Tapsell et al., 2002), Alemania (Fekete, 2009), Latinoamérica (Hahn et al., 2003; Cardona, 2005), Australia (Dwyer et al., 2004) las Filipinas (AcostaMichlik, 2005) o diferentes regiones a nivel mundial (Nakamura et al., 2001).

En lo que se refiere a la cartografía de la vulnerabilidad al riesgo de inundación, su investigación ha ido aumentando significativamente con el paso del tiempo y alcanza ya un alto grado de precisión con metodologías diversas que integran los distintos componentes del riesgo. En Australia (Dwyer et al., 2004); Bélgica (Coninx y Bacus, 2007); Alemania (Fekete, 2009; Sebald, 2010; Scheuer et al., 2011); Italia (Scolobig y De Marchi, 2012); Reino Unido (Alexander et al., 2011; Tapsell et al., 2002); Varios países (Balica et al., 2009). Por su parte, en España se han realizado algunos intentos para evaluar ese tipo de vulnerabilidad, aunque, tal y como señala Perles (2010) y Camarasa y López (2008), el paso hacia la aplicación plausible del concepto a través de la 
elaboración de cartografías se encuentra en su estado más inicial. De hecho, en el último informe CapHaz-Net, que realiza un balance del estado de la cuestión a nivel europeo, solo aparece mencionado el trabajo de Weichselgartner (2002) sobre la evaluación de la vulnerabilidad en algunos municipios de Cantabria. Faltaría mencionar trabajos notables como los de Camarasa (2007, 2008 , 2012) sobre dos cursos del entorno de la ciudad de Valencia; Perles (2010) en Málaga, y Díez et al. (2008) en Castilla-La Mancha, que ofrecen una idea bastante rigurosa de los problemas de vulnerabilidad que afectan a ciertas zonas del ámbito español.

En conjunto, representan una gran variedad de metodologías de diversa naturaleza, con distintas soluciones para la evaluación del fenómeno de la vulnerabilidad. A pesar de todos estos avances que se vienen comentando, existen todavía ciertos retos que, según distintos autores (Adger 2006; Birkman, 2007), desmerecen la precisión de los resultados, lo que hace que la planificación estratégica para la gestión de riesgos sea muy difícil (Tapsell et al., 2010), e incluso termine empeorándola (Cardona, 2001). Uno de ellos es la cuestión de la escala y la disponibilidad de la información correspondiente en cada nivel.

Para, al menos, poner orden en esta cuestión, se han ido proponiendo distintos modelos conceptuales del riesgo que, con mayor o menor éxito, organizan y completan el análisis de la vulnerabilidad a diferentes escalas. Uno de los más conocidos es el de "presure and release» (Blaikie et al. 2014), en el que el riesgo se postula como el resultado de la concurrencia de unas condiciones de vulnerabilidad y de unas posibles amenazas a diferentes escalas. En el presente estudio interesan las condiciones de dicho modelo que acontecen en el nivel local y que se denominan "condiciones de inseguridad». Este tipo de características, entre las que destacan las puramente sociales (genero, raza, etnia, capacidad financiera, nivel de educación, etc.), suelen considerarse a una escala general pese a que los modelos de mayor detalle, según algunos, suelen ser los más importantes para abordar la mitigación del riesgo (Olcina, 2004). Para Fekete et al. (2010) esta cuestión viene explicada por la falta de información y fuentes estadísticas suficientes. Efectivamente, la escasez de información actualizada con ese nivel de detalle supone un obstáculo difícil de salvar para la elaboración de investigaciones de precisión y operatividad efectiva. Hasta el momento, los trabajos más detallados resuelven el problema localizando los datos cuantificables en los censos de población, es decir, aquellos que representan las características intrínsecas de la vulnerabilidad. Sin embargo, simplificando de esta manera, se corre el riesgo de pasar por alto factores fundamentales o casos particulares que prestan gran sentido al valor final de vulnerabilidad social. Una de esas cuestiones serían las relativas a la percepción de la población ante la hipotética presencia de un riesgo, investigación que ya en su día iniciaron Gilbert White, Robert Kates y Ian Burton (Kates, 1962; Burton y Kates, 1964; Burton et al., 1968). La acción o decisión implícita que el concepto de riesgo tiene asociada hace necesaria la relación entre la percepción subjetiva del riesgo y la obligatoriedad científica de su objetivación mediante una evaluación. Conceptual y pragmáticamente es insatisfactorio dejar el asunto como 


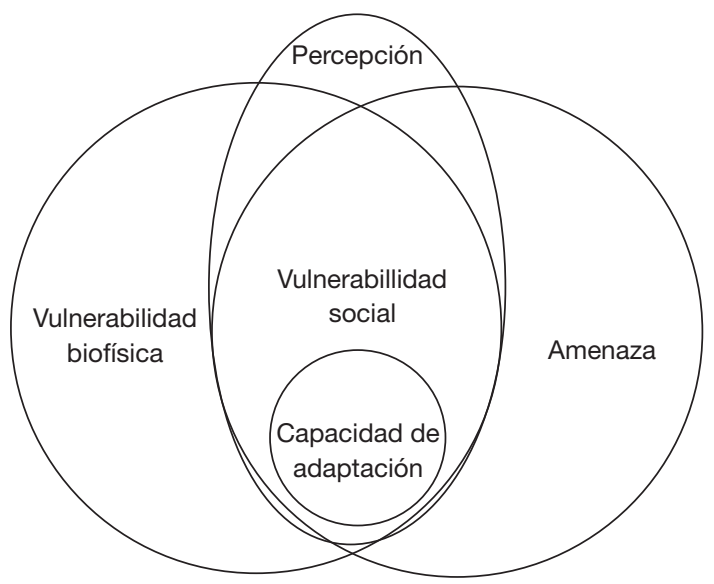

Figura 1. Marco conceptual para la evaluación de la vulnerabilidad social.

Fuente: elaboración propia.

una simple situación relativa y decir que subjetivamente cada persona define y asume el riesgo a su manera, sino todo lo contrario, la percepción constituye un factor más que, junto con los intrínsecos de la social, se confunden e influyen en el estado final de la vulnerabilidad global (véase el trabajo realizado por Lara et al., 2010 y Lara, 2012). En otras palabras, las decisiones que determinan muchas veces la condición socioeconómica o sociodemográfica de un grupo de personas o de un individuo están estrechamente influenciadas, entre otras cosas, por la percepción del riesgo. De hecho, y de acuerdo al presure release, se trata de esas fuerzas o presiones (incluida ahora la ausencia de percepción) las que se acumulan con el paso del tiempo y determinan que un grupo se vea forzado muchas veces a ocupar lugares expuestos $y$, en consecuencia, a padecer los efectos más dañinos de un peligro.

Por tanto, la importancia de la misma es básica como parte de dichas evaluaciones (Cardona, 2001; Tapsell et al., 2005) y debe ser considerada en un modelo conceptual a escala local para la evaluación de la vulnerabilidad social tal y como se ilustra gráficamente en la Figura 1.

El diagrama de Venn de la Figura 1 representa un modelo anidado de características similares al de Smit y Wandel (2006), adaptado a los pormenores señalados de la escala local. Por un lado, estaría la amenaza, y por otro, la vulnerabilidad biofísica, término este último que, según Cutter (1996), habla sobre las características del contexto geográfico del lugar analizado que acentúan o disminuyen los efectos de la amenaza. Por ejemplo, para el caso de las inundaciones, las pendientes, los tipos de suelo, la cobertura vegetal, etc. De la combinación de estos dos elementos, se obtiene un área expuesta que presentará mayores o menores valores de vulnerabilidad social en función de su capacidad de adaptación, de sus valores intrínsecos propios y de la percepción. 
Ésta última representa un elemento que, en términos de Cardona (2001), «convoluciona» o se influye mutuamente con las características sociales y la capacidad de adaptación incluidas en el área con trama rallada. En otras palabras, la percepción ajustada de la realidad permite al individuo tomar decisiones más eficientes que mejoran su condición socioeconómica y sociodemográfica y su capacidad de adaptación, de modo que, en lo que se refiere al riesgo, le mejora su vulnerabilidad social. Según lo anterior, el conjunto de características socioeconómicas, sociodemográficas y la percepción social del riesgo, limitan las capacidades de desarrollo, la capacidad de prevención y la respuesta frente a la materialización de una catástrofe de un grupo de población.

En definitiva, y de acuerdo a todo lo dicho hasta ahora, analizar la conciencia general de un grupo de población sobre la presencia de un peligro es, como así lo indican varios autores (Wachinger, et al., 2010), un factor que debe ser integrado dentro del modelo de evaluación de la vulnerabilidad social, a fin de diagnosticar el valor de la misma en un momento determinado, con el objetivo de facilitar la adopción de estrategias para su mitigación eficiente.

\section{Localización y características del área de estudio}

El ámbito de estudio se localiza en el sureste de la península Ibérica, concretamente, en el sur de la Región de Murcia. La disposición orográfica del mismo presenta forma arqueada de relieves cóncavos hacia el mar que delimitan una cuenca hidrográfica litoral de algo más de $49 \mathrm{~km}^{2}$. En ella se organizan varios sistemas de drenaje que vierten sus aguas directamente al mar en las inmediaciones de la ciudad de Águilas. Destacan las ramblas de Peñaranda, Labradorcico y Renegado-Culebras. Individualizada de las anteriores, la rambla del Cañarete rodea la mitad occidental de la cuenca de Águilas con una red bien jerarquizada (Figura 2). Los factores y elementos climáticos predominantes, la disposición orográfica anteriormente señalada, y la naturaleza impermeable del suelo configuran un tipo de red hidrográfica que experimenta largos periodos de estiaje y que entra solo en funcionamiento en situaciones de lluvia de fuerte intensidad horaria generando crecidas e inundaciones de tipo flash-flood.

Esta escasez e irregularidad de las precipitaciones predominantes en la zona condicionó las primeras etapas de ocupación de estas tierras a través de fuertes lazos de dependencia y respeto hacia los cursos fluviales más importantes. La práctica agrícola con rendimientos regulares y capaz de atender las necesidades mínimas de sus pobladores, se relacionaba, muy directamente, con las posibilidades de aportar a los cultivos agua procedente de esos cauces naturales (Morales, 1968). La distribución de los yacimientos arqueológicos respalda esta conjetura y evidencian una actitud inicial de los primeros ocupantes que se limitó al desarrollo de un conjunto de medidas para aprovechar esos caudales esporádicos y un poblamiento que evitaba el peligro de inundación, localizándose en espacios elevados (Pérez, 2008).

A medida que esa sociedad fue desarrollándose económica y tecnológicamente, se pierde el respeto que durante siglos había permitido eludir con 
Frecuencia de episodios de inundación

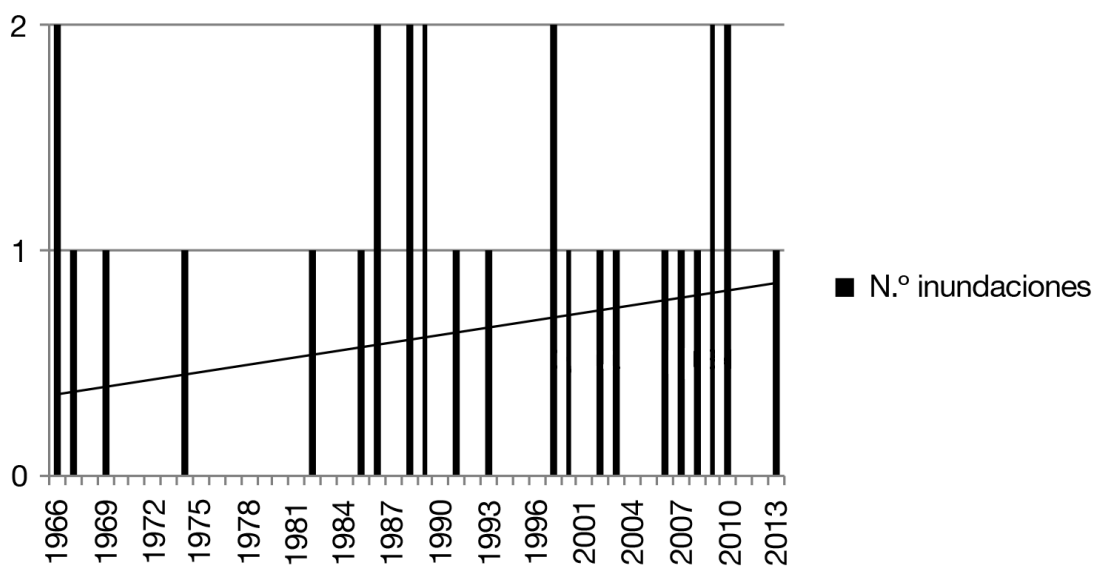

Figura 2. Evolución en la frecuencia de episodios de inundación en el área de estudio (19662013).

Fuente: elaboración propia.

cierta habilidad el problema de las inundaciones, y se produjo un incremento de la exposición acompañado de la frecuencia en el número de catástrofes que afectaron a Águilas (Figura 2). La confianza depositada en unas supuestas capacidades de resistencia y control de la naturaleza motivaron, por un lado, el cambio hacia una agricultura de regadío y altamente transformadora del territorio, el abandono de una gran superficie agrícola dedicada al aprovechamiento de aguas pluviales y, en consecuencia, de su capacidad de amortiguación de la onda de crecida en caso de lluvias de fuerte intensidad horaria. Y por otro, en lo relativo a la evolución urbana (Figura 3) y que, a la postre, supuso la integración forzada de los tramos finales de varias ramblas y ramblizos con los problemas posteriores añadidos que eso conllevó. Este tipo de cambios se acentuó, sobre todo, en las últimas décadas, y una muestra de ellos se evidencia también en los procesos de artificialización registrados en la evolución de los usos de suelo (Tabla 1).

En este contexto más o menos generalizado por todo el Mediterráneo español, cada vez que acontece una inundación de rango extraordinario lo hace de forma catastrófica o, al menos, generando impactos cada vez más intensos (Gil

Tabla 1. Diferencia porcentual según usos entre los años 1996 y 2006

\begin{tabular}{ccccc}
\hline 1990-2006 & Espacio artificial & Espacio agrícola & Terrenos regados & Espacio natural \\
\hline Diferencia (ha) & $+195,05 \%$ & $+98,43 \%$ & $+203,13 \%$ & $-293,48 \%$ \\
\hline
\end{tabular}

Fuente: Corine Land Cover 96 y 06. 


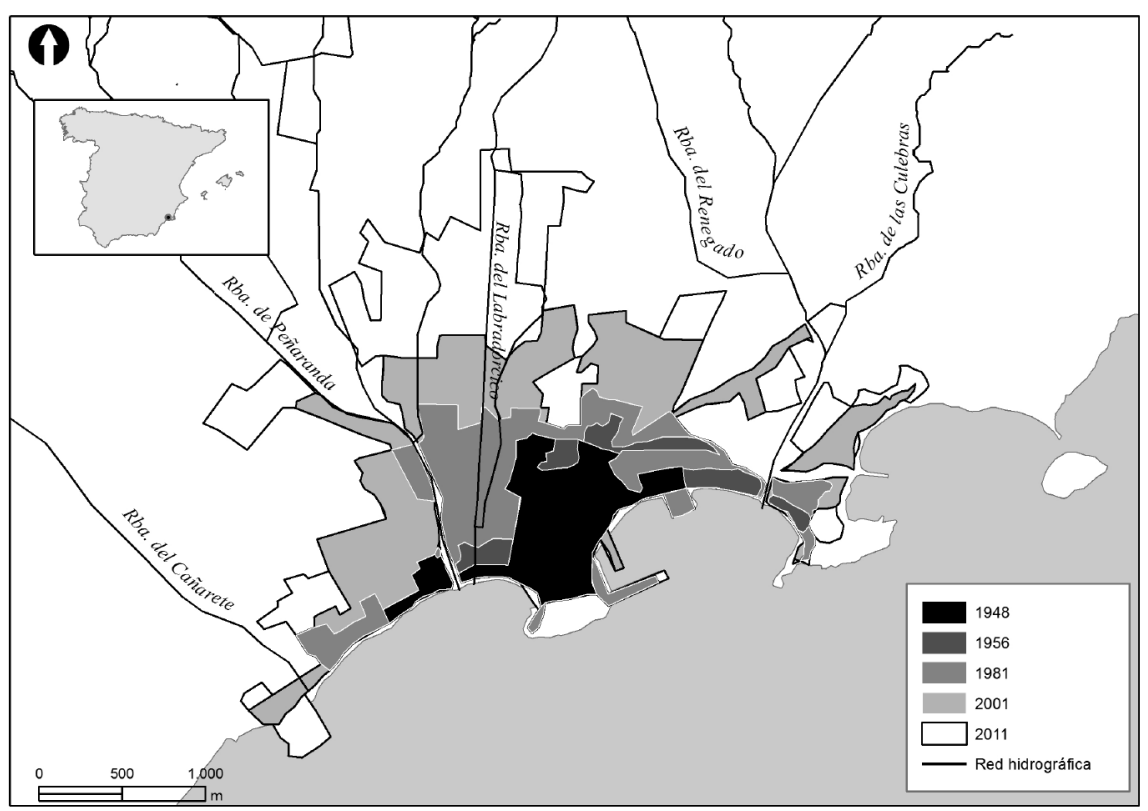

Figura 3. Mapa de localización de la ciudad de Águilas y crecimiento del núcleo urbano entre 1948 y 2011.

Fuente: elaboración propia.

et al., 2014). Ante esta situación, la población suele reaccionar demandando de modo apremiante la implantación de actuaciones estructurales para mitigar los problemas crecientes de las avenidas. Sin embargo, eso apenas supone un parche al mismo, pues la exposición al riesgo, en la mayoría de los casos, va por delante de los umbrales teóricos de seguridad que establecen dichas medidas defensivas. Ante este conflicto, las estrategias de carácter preventivo como la ordenación el territorio, bien enfocadas, se convierten en un instrumento eficaz para, entre otras cosas mitigar el riesgo de inundación. Lamentablemente, la aplicación que se viene haciendo de las mismas en el área de estudio destaca por sus carencias en la forma de contemplar el riesgo de inundación (Pérez, 2008), sobre todo por la tendencia de dichas medidas de rentabilizar al máximo el uso del territorio en forma de procesos de ocupación.

En esta secuencia de pasos desafortunados, la población es la que finalmente acaba por experimentar los mayores efectos y es, sobre todo, la más débil y desfavorecida la que achaca de forma más acusada los impactos de estos fenómenos naturales. En consecuencia, resulta interesante, como paso inicial, centrar los esfuerzos en el análisis e identificación de esos sectores de población a fin de mejorar, principalmente, las deficiencias señaladas en materia de ordenación y/o planificación. 


\section{Objetivo y metodología}

El objetivo del presente trabajo es el de integrar los principales indicadores sociales (sociodemográficos y socioeconómicos) más comúnmente utilizados junto con la percepción, en un índice sintético destinado al diagnóstico del comportamiento de variables sociales indicadoras de vulnerabilidad frente a inundación en una zona inundable. Al tratarse de un estudio de alta resolución espacial, se ha considerado llamar dicho índice como INVUSOL (Índice de Vulnerabilidad Social Local).

Para la construcción estadística del índice y su posterior representación cartográfica se siguió el método aplicado por Cutter et al. (2003), con ciertas variaciones dadas las limitaciones propias derivadas de la disponibilidad de información y la consideración de otros factores (como la percepción) que no se tuvieron en cuenta en dicho estudio:

1. Delimitación de la exposición para identificar las secciones censales afectadas en caso de inundación. Para ello se combinó el mapa de secciones censales con la modelización hidrológica del Periodo de Retorno de 500 años, contenida en el Sistema Nacional de Cartografía de Zonas Inundables.

2. Una vez recolectados los datos procedentes del Censo de población y viviendas del INE (2001) se normalizaron todas las variables en porcentajes.

3. Se verificó el conjunto de datos empleando estadísticas del tipo descriptivo (mín./máx., media, desviación típica) a fin de localizar valores perdidos.

4. Se tipificaron las variables obtenidas $\frac{\chi-\mu}{\sigma}$. Este procedimiento da como resultado variables con media 0 y desviación típica 1.

5. Se analizó la multicolinealidad a fin de distinguir aquellas variables que podrían ser redundantes dentro del modelo explicativo. Las variables eliminadas fueron cuatro. Esto es debido a que sus valores en la diagonal principal de la matriz de correlaciones, las señalaban como las de menor valor de medida de adecuación muestral al presentar valores de correlación altos con respecto al resto y, por tanto, innecesarias.

6. Como paso previo a la aplicación del análisis de componentes principales (ACP) se calculó el test KMO $(0,652)$ y la prueba de esfericidad de Barlett (p-valor) $<0,05$. Ambos resultados indican que se podía aplicar el ACP.

7. Se llevó a cabo el ACP usando la rotación varimax y el criterio Kaiser para la selección de componentes. El gráfico de sedimentación resultante permitió determinar los factores ocultos entre el grupo de variables que, de forma conjunta, explican la mayor parte de la variabilidad del valor de vulnerabilidad. Se seleccionaron los componentes con carga superior a 1 y se sintetizó la información en tres nuevos factores que más adelante se comentan.

8. Se tipificó el resultado definitivo de la suma de factores y se añadió el valor de percepción (F4), previamente estandarizado, siguiendo la siguiente fórmula:

$$
I N V U S O L=F 1+F 2+F 3+F 4
$$


El valor final sirve para determinar el grado de vulnerabilidad a nivel de sección censal.

9. La confección de la cartografía de riesgo siguió el esquema de la Figura 4. Mediante su aplicación se logra evidenciar las zonas expuestas a inundación y el grado de vulnerabilidad social que presentan a dicho peligro. La representación del valor del INVUSOL se hizo mediante el método de las desviaciones típicas.

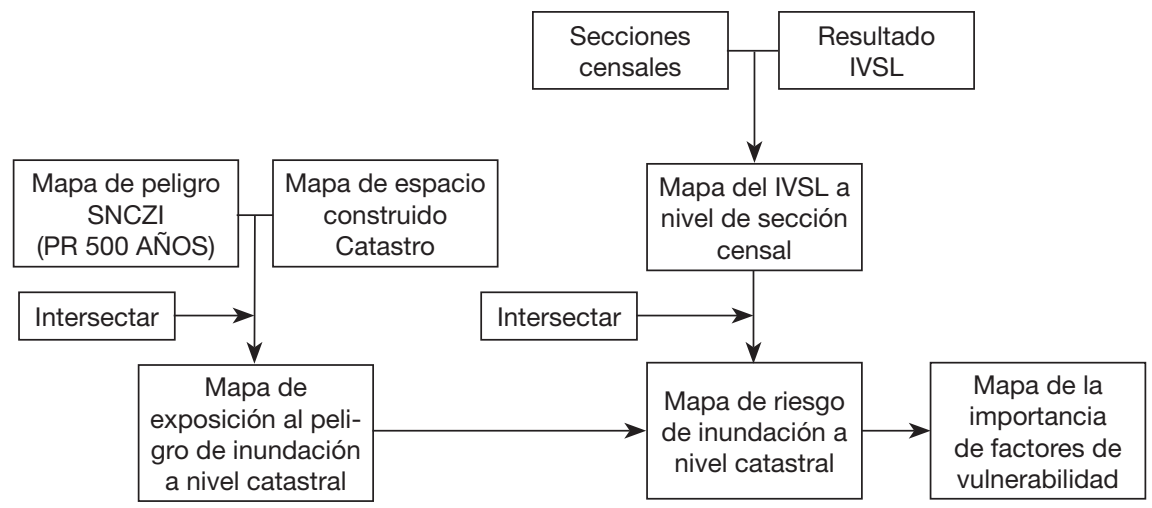

Figura 4. Organigrama del proceso de elaboración cartográfico.

Fuente: elaboración propia.

10. Finalmente, se llevó a cabo un segundo mapa donde se clasifican las secciones censales y los inmuebles contenidos en las mismas a partir del análisis estadístico de los resultados del INVUSOL. El valor de cada factor superior a la media más una desviación típica se convierte en una categoría que permite identificar la sección que destaca en dicho factor.

\section{Procedimiento para la construcción del índice de vulnerabilidad social y su representación cartográfica}

\subsection{Delimitación de la exposición}

El punto de partida es el conocimiento de los ámbitos potencialmente afectados por un peligro, los cuales se identifican mediante la cartografía de exposición (Figura 5). La información necesaria para la realización de la misma proviene de una fuente que proporciona la superficie inundable para el periodo de retorno de 500 años, el SNCZI (Sistema Nacional de Cartografía de Zonas Inundables). Se trata del instrumento oficial elaborado por el Ministerio de Agricultura, Alimentación y Medio Ambiente que, siguiendo los principios de la Directiva 2007/60/CE sobre evaluación y gestión de riesgos de inundación, se emplea en la gestión del espacio fluvial, la prevención de riesgos y la planificación territorial. 


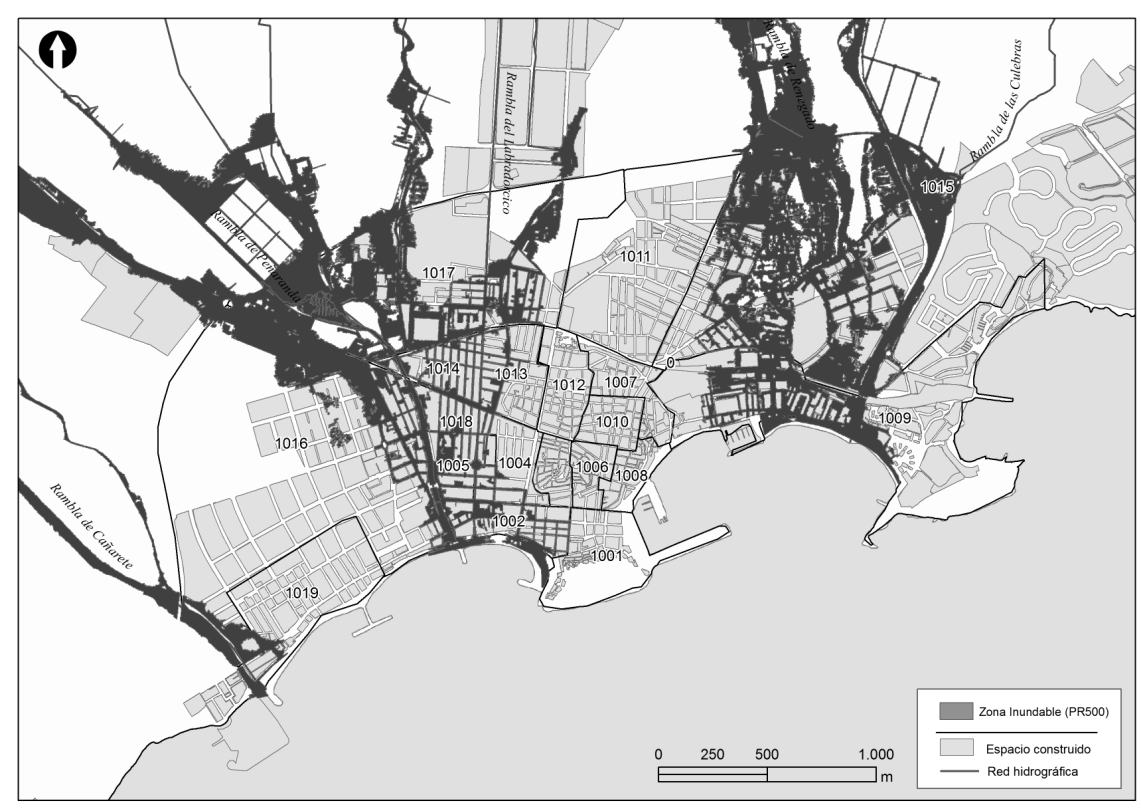

Figura 5. Mapa de exposición compuesto por el resultado de la modelización del SNCZI (PR500) y las secciones censales.

Fuente: elaboración propia.

El análisis de la peligrosidad debe completarse con el estudio de la distribución de la población a fin de localizar el ámbito espacial expuesto, considerando que el emplazamiento concreto tiene una gran relevancia respecto a la intensidad del previsible impacto. En este sentido, se ha llevado a cabo una doble delimitación a diferente escala y en función del cometido de la misma. En primer lugar, se seleccionaron las secciones censales inundadas para el periodo de retorno de 500 años. Esta delimitación previa resultó útil para el análisis estadístico de los factores de vulnerabilidad que definen a dichas secciones censales. Sin embargo, sus límites administrativos sobrepasan el espacio inundado y carecen de la precisión necesaria para una cartografía efectiva que pueda ser aplicada en tareas de mitigación de la vulnerabilidad. Para solventar este problema, la información catastral resulta especialmente valiosa, pues facilita la localización de las edificaciones que se sitúan en una sección afectada por las inundaciones. La base cartográfica del catastro nacional (<www.sedecatastro.gob.es $>$ ) proporciona las parcelas urbanas que, una vez intersectadas con la zona inundable, permiten identificar aquellas que están expuestas y, posteriormente, asociar el valor final de vulnerabilidad a las mismas para ser representado cartográficamente.

Pese a ese aumento del grado de precisión comentado, hay que señalar la debilidad metodológica que supone generalizar el resultado del índice de vulnerabilidad a una porción de una sección representado por parcelas catastrales. 
En ocasiones, el error de esa generalización podría ser abultado; sin embargo, el secreto estadístico no permite depurar dicha cuestión y hay que conformarse con ese resultado.

\subsection{Cálculo de la vulnerabilidad social}

Fijada la exposición, se abordan los elementos que generan una situación de desventaja o debilidad estructural propia del grupo humano. Aquellos colectivos que concentran el mayor número de factores genéricos de vulnerabilidad - pobreza, dependencia, desconocimiento del medio geográfico, ignorancia de sus derechos, falta de capacidades—, son también los que muestran mayor indefensión, en el sentido de no poder contar ni con protección ni con todos los apoyos necesarios para evitar el peligro o luchar contra él. Como ya se ha indicado, la caracterización de la vulnerabilidad social frente a inundaciones se ha realizado mediante la integración, por un lado, de una serie de indicadores que dan cuenta de aspectos sociodemográficos y socioeconómicos, y por el otro, de los resultados obtenidos en una encuesta de percepción.

La información necesaria para organizar los primeros indicadores se obtuvo del censo de población y viviendas de 2001 del INE ${ }^{1}$. La selección de los mismos se realizó según una extensa revisión bibliográfica y de acuerdo a los criterios de Dwyer et al. (2004), es decir: validez, disponibilidad, calidad de la información, precisión, simplicidad, notoriedad científica y objetividad. Una vez efectuado ese primer escrutinio, la justificación de cada una de estas variables intervinientes en el resultado final de vulnerabilidad, se entiende de la siguiente manera:

a) Población con respecto al total del área de estudio (POB). La población total es un indicador descriptivo que ofrece una idea general de la composición demográfica del área de estudio. El número de personas potencialmente expuestas es un dato que ayuda a distinguir aquellos lugares que, independientemente de sus características sociales, presentan un mayor o menor volumen de efectivos, potencialmente afectables, lo que en última instancia acentúa o disminuye el grado de vulnerabilidad de la zona.

b) Tasa de feminidad (FEM). Las mujeres pueden experimentar un periodo de recuperación mayor que los hombres debido al grado de especialización de género en sectores específicos, a los salarios más bajos, y a las responsabilidades tradicionales del cuidado de las familias.

c) Población mayor de 65 años $(\mathrm{POB}>65)$. Las personas de edad avanzada pueden presentar limitaciones de movilidad y dependencia que reducen su capacidad de recuperación tras la materialización de una catástrofe.

d) Población infantil menor de 4 años $(\mathrm{POB}<4)$. De igual forma que el grupo anterior, las edades tempranas llevan implícito un grado de dependencia

1. Se han empleado los datos del censo de 2001 dado que los de 2011 no están abiertos al público por secreto estadístico, o porque el censo cuenta con una muestra demasiado pequeña del ámbito analizado como para asegurar la precisión de los mismos. 
y dificultades de movimientos debido a su condición física. Los desastres por inundaciones suponen situaciones muy difíciles para estas personas no solo la dificultad para moverse rápidamente, si no también, por su desconocimiento absoluto de una circunstancia crítica y compleja como es una inundación.

e) Hogares unipersonales $(\mathrm{HOG}>64)$. El grupo de personas de la tercera edad que viven solos suelen tener serias limitaciones para superar situaciones de crisis que desestabilizan su equilibrio socioeconómico. Cuando una amenaza natural del tipo inundación afecta a sus viviendas o a este tipo de personas, su grado de dependencia se acentúa sobremanera y se enfrentan a circunstancias que en muchos casos son insalvables por sus propios medios.

f) Hogares con un adulto y un menor o más (HOGMON). Las familias monoparentales con altos números de dependientes muchas veces tienen una capacidad financiera limitada y no pueden contratar servicios de atención de esos menores, por lo que deben compaginar las responsabilidades familiares con las profesionales. Todo lo anterior afecta a la capacidad de recuperación tras un desastre.

g) Población extranjera (EXT). Por lo general, el número de individuos extranjeros contribuye al aumento de la vulnerabilidad social a través de su dificultad de acceso a recursos, la diferencia cultural y la marginación social, económica y política. Sin embargo, en el caso del área de estudio se debe distinguir dos grupos bien diferenciados en función de su procedencia y su capacidad de financiación. Por un lado, el colectivo social de Centroeuropa, con un poder adquisitivo alto que se instalan en el litoral mediterráneo sin otra pretensión que disfrutar de las condiciones climáticas de la zona. A pesar de ello, se trata de un colectivo especialmente vulnerable al tratarse en su mayor parte de personas mayores con una reducida percepción de la peligrosidad latente en el medio que habitan. Por otro, están el grupo de inmigrantes africanos y suramericanos cuyas características se ajustan a las tradicionales ya señaladas, por las cuales se considera vulnerables a los extranjeros.

h) Población extranjera infantil (EXTINF). La condición de extranjero comentada en el anterior indicador se agrava cuando dicha población es de edad infantil. Se trata de individuos poblacionales muy sensibles que padecen situaciones de desamparo cuando acontece una catástrofe.

i) Población en paro (PARO). El desempleo limita seriamente las capacidades financieras de la población y dificulta en gran medida sus posibilidades de recuperación, lo que en última instancia agrava su vulnerabilidad. Además, el problema suele acentuarse tras una situación de crisis, ya que los efectos de la misma pueden destruir las formas de producción que dan trabajo a esa población.

j) Población juvenil en paro (PAROJU). La población en paro del tipo juvenil presenta el agravante de la edad en cuanto a sus posibilidades de responder ante una situación difícil derivada de la materialización de un peligro natural. 
k) Ocupados eventuales (OCUEV). Las características de temporalidad en la población que desempeña un trabajo, influyen negativamente sobre la estabilidad económica de la misma. Los individuos que se enfrentan a estas circunstancias poseen serias dificultades de afrontar los efectos negativos de una riada durante el periodo de recuperación.

l) Ocupados no cualificados (OCUNC). Las personas no cualificadas son el colectivo más susceptible de perder su empleo ante cualquier tipo de eventualidad, ya sea de origen natural o económico. Para el caso de las inundaciones, la posible destrucción de las formas productivas por el efecto del agua, suele venir asociado con un incremento puntual del número de despidos en las empresas afectadas que se ceba de forma especial con el grupo comentado.

m) Población sin estudios (ANALF). El nivel de formación educativo está vinculado con la consecución de unos ingresos más altos. El hecho de tener menos educación obstruye incluso la capacidad de comprensión de la información de advertencia y de acceso a la recuperación.

n) Población discapacitada (DISCA). La población discapacitada es uno de los colectivos que puede verse más afectado por su evidente condición de desventaja física con respecto al resto de la sociedad. Este grupo de personas son enteramente dependientes de otras y requieren la atención continua de especialistas en ciertos casos. En situaciones de emergencia o catástrofe, su evacuación se dificulta en gran medida y durante el periodo de recuperación padecen más que cualquier otro las posibles afecciones a sus viviendas y/o lugares de residencia.

\subsection{Percepción}

De acuerdo al marco conceptual propuesto, la medición concreta de la vulnerabilidad social se debe completar con la evaluación de la actitud de una población ante el riesgo. Esto exige un amplio trabajo de campo, de índole multidisciplinar y, en particular, la aplicación de encuestas y entrevistas. Ya se propusieron, en su día, estos procedimientos para medir la percepción de algunos riesgos entre grupos potencialmente afectados (Burton y Kates, 1964). No obstante, parece necesario recabar información más amplia que incluya el conocimiento de los problemas generales del grupo y el papel relativo que dentro de estos asignan a la existencia de riesgo. Es conveniente saber cómo evalúan los afectados potenciales las acciones que emanan de los distintos niveles de la Administración, así como la descripción y la valoración de su propia acción individual, familiar o de unidad de convivencia, y también conocer su opinión y valoración respecto a futuras actuaciones en el caso de que existan.

Para profundizar en esta cuestión, se seleccionaron y analizaron las encuestas realizadas en un proyecto de investigación previo del que derivó, entre otros, un trabajo de Calvo y Granell (2009) donde se profundiza en el diseño de la muestra. Basta mencionar que el muestreo fue probabilístico estratificado con selección de conglomerados. La encuesta, basada en un guion previamente 
establecido, se realizó en la primavera-verano de 2008 sobre las secciones censales del núcleo urbano de Águilas potencialmente inundables por el periodo de retorno de 500 años.

En total, se sondeó una muestra de 350 encuestas sobre una población de 19.817 habitantes (2008) localizada en cuatro zonas inundadas por el periodo de retorno de 500 años. La muestra se calculó con un margen de error del 5,19\% y un nivel de confianza del $95 \%$. De modo preferente, se seleccionaron personas de cuarenta y cinco años o más $(60,1 \%)$ y residentes habituales en el área (90\%). Considerando que los episodios de inundación catastrófica que se produjeron en el área de trabajo hasta la fecha de la realización del sondeo se concentraron principalmente en las décadas de los setenta a noventa del siglo pasado, parece que este segmento de población fue el más adecuado para aportar su valoración. Cabe señalar, sin embargo, que la presencia de residentes turísticos europeos que ocupan segundas residencias en el litoral, así como de numerosos trabajadores de origen magrebí o subsahariano aconseja atender a este colectivo, especialmente teniendo en cuenta que las víctimas mortales en los últimos episodios de inundación catastrófica han sido exclusivamente extranjeros residentes temporales. Resultado de esta selección en la muestra entrevistada es que un $52,4 \%$ reconoce haber sufrido la experiencia directa de alguna inundación, lo que a los efectos buscados resulta satisfactorio. Las características generales de la población encuestada se expresan en la Tabla 2.

El cuestionario (Tabla 3) se articuló en torno a cuatro dimensiones principales: 1) cuándo y por qué se producen las inundaciones en el sector analizado; 2) cuáles son las posibles causas que motivan las inundaciones; 3) qué opinión merece la gestión por parte de los organismos con responsabilidades en el tema, y 4) la valoración de las medidas adoptadas antes, durante y después de las inundaciones. A efectos del estudio, el análisis de percepción se simplificó en seis cuestiones que revelan, de forma significativa, la opinión de la población en cada uno de los temas anteriormente indicados.

Tabla 2. Descriptores básicos de la población encuestada

\begin{tabular}{llc}
\hline & Descriptores & Águilas \\
\hline Sexo & Hombres & 170 \\
& Mujeres & 180 \\
& Total & 350 \\
\hline Edad (años) & $<25$ & 63 \\
& $25-45$ & 76 \\
& $45-65$ & 156 \\
& $>65$ & 55 \\
\hline Residencia habitual & Esta & 315 \\
& Otra & 35 \\
\hline Afectados & & 199 \\
\hline
\end{tabular}

Fuente: elaboración propia. 
Tabla 3. Cuestionario realizado a la población

\begin{tabular}{|c|c|c|c|}
\hline Dimensiones & Preguntas & Respuestas & Valores \\
\hline \multirow[t]{2}{*}{ 1a } & \multirow[t]{2}{*}{ ¿Se acuerda de la última inundación? } & Sí & 0 \\
\hline & & No & 1 \\
\hline \multirow[t]{2}{*}{$1 \mathrm{~b}$} & \multirow{2}{*}{$\begin{array}{l}\text { ¿Se acuerda de la última inundación después } \\
\text { de } 1980 ?\end{array}$} & Sí & 0 \\
\hline & & No & 1 \\
\hline \multirow[t]{2}{*}{2} & \multirow{2}{*}{$\begin{array}{l}\text { Antes de empezar la inundación ¿estuvo usted } \\
\text { convencido de que algo serio iba a ocurrir? }\end{array}$} & Sí & 0 \\
\hline & & No & 1 \\
\hline \multirow[t]{6}{*}{3} & \multirow{6}{*}{$\begin{array}{l}\text { ¿Cuáles cree Vd. Que son las causas } \\
\text { principales de las inundaciones que sufre } \\
\text { su localidad? (Única respuesta) }\end{array}$} & a. Inadecuada ubicación de la vivienda & 0 \\
\hline & & b. Inadecuada infraestructura de protección & 1 \\
\hline & & c. Razones puramente naturales & 1 \\
\hline & & d. Pobreza de las personas & 1 \\
\hline & & e. Falta de previsión por parte del Gobierno & 1 \\
\hline & & f. Alteración del medio ambiente y cambio climático & 1 \\
\hline \multirow[t]{9}{*}{4} & \multirow{9}{*}{$\begin{array}{l}\text { ¿Qué deberían hacer las autoridades para } \\
\text { evitar un futuro desastre? (Única respuesta) }\end{array}$} & a. Más información & 1 \\
\hline & & b. Mantenimiento de cauces & 1 \\
\hline & & c. Plantar árboles & 1 \\
\hline & & d. Mantenimiento de desagües & 1 \\
\hline & & e. Aplicar un adecuado planeamiento urbanístico & 0 \\
\hline & & f. Agilizar las evacuaciones & 1 \\
\hline & & g. Mejorar los sistemas de prevención y aviso & 1 \\
\hline & & h. Realizar estudios de cauces & 1 \\
\hline & & i. No se puede hacer nada & 1 \\
\hline \multirow[t]{2}{*}{5} & \multirow{2}{*}{$\begin{array}{l}\text { ¿Cree usted que ocurrirá en su localidad otra } \\
\text { inundación en el futuro? }\end{array}$} & Sí & 0 \\
\hline & & No & 1 \\
\hline
\end{tabular}

Fuente: elaboración propia.

El cuestionario sigue un modelo de acierto/error en el que las respuestas que revelan una conciencia de la presencia de un riesgo y de sus efectos potenciales, se interpretan como un acierto, e indica que el colectivo sondeado no es vulnerable en ese sentido. Las respuestas se van sumando y, posteriormente, se normalizan según porcentajes con respecto al total de individuos encuestados. De ese modo, se obtiene un balance general por zonas encuestadas expuestas que se expresa matemáticamente con la siguiente fórmula:

$$
V y=\sum_{i=1}^{n=6} x_{i v / n}
$$

Donde $x$ : respuesta errónea; $i$ : pregunta; $y$ : sección censal encuestada; $V$ : valor de percepción de la unidad encuestada.

Establecidas las bases del cuestionario, el problema se planteó a la hora de determinar la ponderación que se le ha de asignar a cada una de las preguntas para obtener el resultado final preciso de percepción. Se trata de una cuestión de gran complejidad (Zahran et al., 2008) que al igual que Masgrau y Ribas 
(2012), se llegó a la conclusión de que la solución más adecuada era no asignar ningún tipo de peso a los resultados finales del cuestionario. De esta forma se obtiene un modelo simplificado que proporcionó una visión del fenómeno estudiado lo suficientemente adecuada como para ser implementado en el índice de vulnerabilidad social final.

En resumen, los valores de percepción pueden tomar un valor mínimo de 0 y un máximo de 6 , que son el total de preguntas del cuestionario. El valor máximo corresponde a aquellos lugares donde el número de respuestas erróneas es más frecuente, o lo que es lo mismo, donde el grado de conciencia que tiene ese grupo de población ante la presencia de una amenaza es menor con respecto a otros grupos que demuestran estar más preparados ante cualquier tipo de evento de estas características.

\section{Resultados y discusión}

A fin de simplificar los resultados, se ha creído conveniente comentar los resultados parciales según todos los elementos que constituyen el mapa final de riesgo.

En lo referente a la delimitación de la exposición, de un total de parcelas catastrales en la ciudad de Águilas asociadas a edificios de 6.422, $1.617(25,1 \%)$ estarían expuestas a la de riada con periodo de retorno de 500 años. Por su parte, de las secciones analizadas, destacan por su nivel de afección en caso de catástrofe, la 1014 y la 1017, con un número de parcelas urbanas potencialmente afectadas de 222 y 186, y una población de 1.150 y 1.228 habitantes, respectivamente (ver Tabla anexa). Se trata de las secciones más habitadas y que, por su posición en la zona de confluencia tradicional de la rambla de Peñaranda y Labradorcico son las más expuestas al peligro.

Interesaba entonces el cálculo de la vulnerabilidad por secciones para identificar las que contaban con un mayor valor. De la aplicación del ACP sobre las variables socioeconómicas y sociodemográficas, se obtuvieron los resultados que se resumen de acuerdo a la Tabla 4. Estos indican que fueron tres los factores principales que estaban ocultos en dicho conjunto de indicadores: distribución de la población, estructura demográfica y estructura socioeconómica. Entre todos ellos explican un total del $81 \%$ de la varianza. Sin embargo, aunque el modelo explicativo parece completo, e incluso mejorado con la consideración posterior de factor percepción, cabe preguntarse si dicho análisis resultaría más preciso si se superaran las limitaciones relativas a la recolección de información ya señaladas en su día por Kaztman (2000). Efectivamente, muchos de los indicadores necesarios para cuantificar y explicar la varianza de la variable dependiente, en este caso, la vulnerabilidad social, no están disponibles, o si lo están, se encuentran a una escala que no resulta útil para su aplicación en estudios como el que interesa o, en ocasiones, muy desactualizados (Gall et al., 2009). A pesar de las mencionadas limitaciones, los factores resultantes (Tabla 4) se aproximan a los obtenidos en trabajos previos (Cutter 
Tabla 4. Dimensiones de la vulnerabilidad social tras el ACP

\begin{tabular}{clclc}
\hline Factor & \multicolumn{1}{c}{ Nombre } & $\begin{array}{c}\text { Porcentaje de } \\
\text { varianza explicado }\end{array}$ & \multicolumn{1}{c}{$\begin{array}{c}\text { Variable } \\
\text { dominante }\end{array}$} & Correlación \\
\hline 1 & Distribución de la población & 43,11 & Población & $+0,91$ \\
2 & Estructura demográfica & 27,33 & Población infantil & $+0,83$ \\
3 & Estructura socioeconómica & 10,57 & Población en paro & $+0,93$ \\
\hline
\end{tabular}

Fuente: elaboración propia.

et al., 2003; Holand y Lujala, 2013) aunque difieren en número y orden de importancia debido a las desigualdades de obtención de información en las fuentes estadísticas consultadas.

El primer factor es el más heterogéneo y se llamó «distribución de la población». En él se engloban el porcentaje de población total, la población discapacitada y los hogares unipersonales compuestos por una persona mayor de 64 años. Principalmente, los dos primeros, presentan una estrecha dependencia dada la proporcionalidad que se ha detectado en cuanto al número de personas discapacitadas en relación al de población residente. Por su parte, los hogares habitados por un único adulto tienen una correlación negativa con respecto al factor, dado que se registran en menor número donde más población se concentra. Se trata de un comportamiento propio de una ciudad cuyo modelo de crecimiento viene condicionado por el turismo. La población de mayor edad se distribuye alrededor del núcleo principal de población o en el centro y se puede clasificar en dos grupos: los nacionales y, sobre todo, los extranjeros. Este colectivo, muy numeroso en el caso de Águilas, tiende a localizarse en las secciones censales periféricas de la ciudad, más próximas a la primera línea de playa o en zonas de nueva expansión, como así sucede en el caso estudiado.

El segundo factor hace referencia concreta a los indicadores relacionados con la estructura de la demografia analizada, principalmente, en lo que concierne a la edad. En él se incluye la población infantil ( $<4$ años) y adulta $(>65$ años) además de los hogares compuestos por un adulto y un menor o más. Tal y como señalan autores como Cutter, et al. (2000); Mileti y O'Brien (1992); Hewitt (1997) y Ngo (2001), estos grupos de edad son los más afectados por los desastres. La presencia de este tipo de habitantes es señal inequívoca de un alto grado de vulnerabilidad.

En el tercer factor, estructura socioeconómica, se incluye la población en paro, población en paro juvenil y ocupados no cualificados. Las anteriores variables presentan una homogeneidad evidente en cuanto a los indicadores que la configuran y que están en sintonía con lo ya señalado en otros trabajos como (Heinz Center for Science, 2000; Hewitt 1997; Puente, 1999 y Dwyer et al., 2004). En consecuencia, las secciones censales caracterizadas por esta base de empleo podrían sufrir un mayor impacto de los peligros naturales y afrontar una recuperación más lenta tras un desastre.

En cuanto a los resultados de percepción inferidos de las encuestas (que, para el presente trabajo, constituye el cuarto factor dentro del modelo expli- 
Tabla 5. Resultados estandarizados de cada uno de los factores que contribuyen a la construcción del INVUSOL

\begin{tabular}{lrrrrrl}
\hline $\begin{array}{c}\text { Sección } \\
\text { censal }\end{array}$ & \multicolumn{1}{c}{ F1 } & \multicolumn{1}{c}{ F2 } & \multicolumn{1}{c}{ F3 } & \multicolumn{1}{c}{ F4 } & INVUS0L & \multicolumn{1}{c}{$\begin{array}{c}\text { Factor } \\
\text { destacado }\end{array}$} \\
\hline 1002 & $-0,19429$ & 0,85938 & 0,84586 & $-1,14810994$ & 0,36284006 & Equilibrado \\
1004 & $-0,96131$ & $-0,53513$ & $-0,49263$ & $-1,14810994$ & $-3,13717994$ & Equilibrado \\
1005 & 0,74902 & $-0,23365$ & 0,34578 & $-1,14810994$ & $-0,28695994$ & F1 \\
1009 & 0,32177 & $-0,33721$ & $-0,54718$ & $-0,37735082$ & $-0,93997082$ & Equilibrado \\
1011 & $-1,45978$ & $-1,34095$ & 0,01371 & 1,19628238 & $-1,59073762$ & F4 \\
1013 & $-0,35604$ & $-1,03889$ & $-0,75704$ & $-1,14810994$ & $-3,30007994$ & Equilibrado \\
1014 & 0,04592 & $-1,0061$ & $-0,37543$ & $-1,14810994$ & $-2,48371994$ & Equilibrado \\
1015 & $-1,76758$ & 2,81019 & $-0,32883$ & $-0,37735082$ & 0,33642918 & F2 \\
1016 & $-1,60561$ & $-0,81696$ & 0,68801 & 0,32917837 & $-1,40538163$ & Equilibrado \\
1017 & 0,47092 & 0,67904 & 2,28835 & 1,19628238 & 4,63459238 & F3 y F4 \\
1018 & 0,23857 & $-0,40057$ & 1,31553 & $-1,14810994$ & 0,00542006 & Equilibrado \\
1019 & 0,13477 & $-0,7639$ & 1,68415 & 0,32917837 & 1,38419837 & F3 \\
Media & $-0,36530333$ & $-0,1770625$ & 0,39002333 & $-0,38270331$ & $-0,53504581$ & \\
DT & 0,86781186 & 1,14718431 & 0,98400726 & 0,93005786 & 2,18627444 & \\
M+Dt & 0,50250853 & 0,97012181 & 1,37403059 & 0,54735455 & & \\
\hline
\end{tabular}

Fuente: elaboración propia.

cativo y de evaluación del índice), los valores presentan una media de $-0,38$, una desviación típica de 0,93 , un valor máximo tipificado de 1,19 y mínimo de $-1,14$. Los más bajos se corresponden con la zona norte de la ciudad, precisamente el sector más deprimido y con mayores problemas de desigualdad e integración con el resto del espacio urbano (ver Tabla 5).

Por el contrario, los valores de mayor conciencia se computan en el centro de la ciudad, en las secciones localizadas en el área inundable de la rambla de Peñaranda. Se trata de un colectivo social que debido a su condición habitacional próxima a un cauce bien canalizado y especialmente activo, ostenta niveles altos de percepción con respecto a sus vecinos dada la influencia física que comporta la presencia del mismo en el entorno de sus viviendas.

Como ya se ha señalado con anterioridad, de la integración de todos estos resultados comentados hasta el momento, se elaboró y cartografió el INVUSOL. Para simplificar la comprensión de los resultados obtenidos se elaboraron dos mapas relativos al valor final de vulnerabilidad. El primero (Figura 6) es el mapa de vulnerabilidad social ante el riesgo de inundación. Los valores de vulnerabilidad de las secciones de Águilas presentan niveles que van de $-4,63$ (vulnerabilidad social alta) a $-2,48$ (vulnerabilidad social baja), con media $-0,53$ y desviación típica 2,18 . La zona que presenta unos valores más elevados de vulnerabilidad corresponde a las secciones censales localizadas en el norte de la ciudad. Tal y como se ha señalado, se trata de uno de los lugares donde los escenarios de marginación y desigualdad son más 


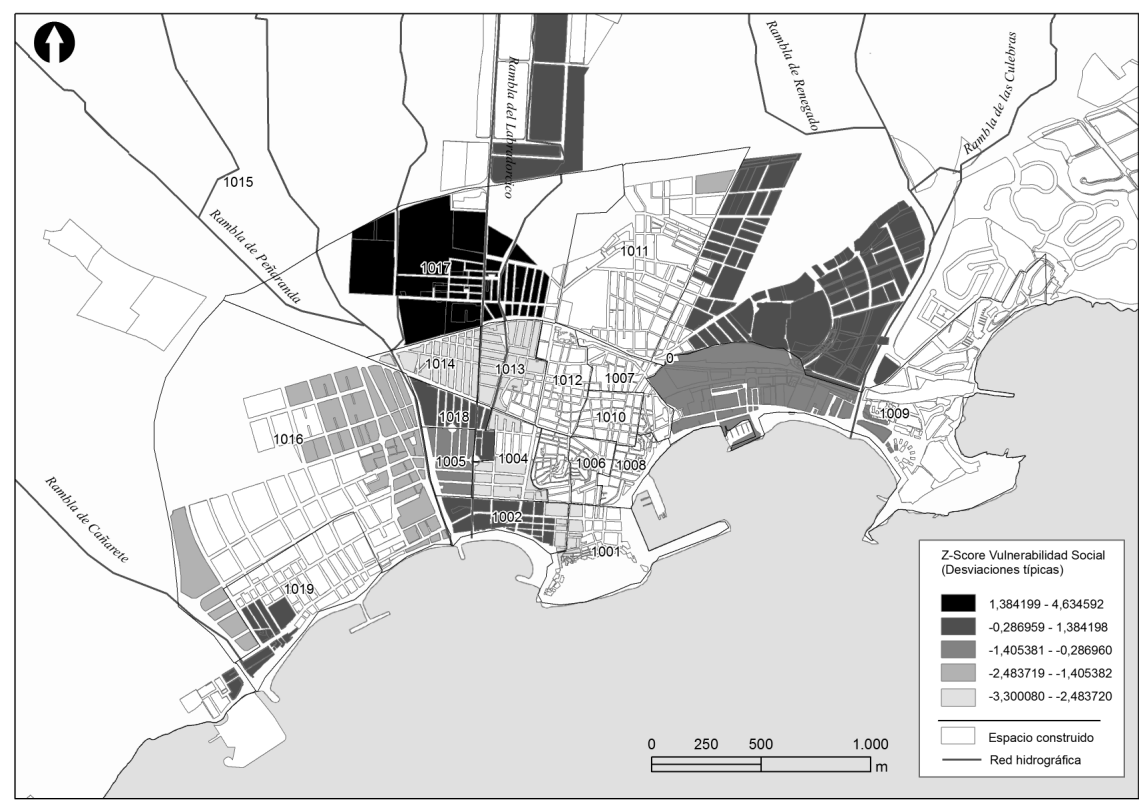

Figura 6. Mapa de vulnerabilidad social ante el riesgo de inundación con las secciones censales.

Fuente: elaboración propia.

evidentes. En ella están comprendidas las secciones censales 1017 y 1011, una población total de 4.134 habitantes y un número de inmuebles potencialmente afectables de 2.609 .

Por último, de acuerdo al mapa de significación por factores (Figura 7), en esas secciones destacan los de la estructura demográfica y, sobre todo, el factor de la percepción. La existencia de un riesgo latente evidenciado por las inundaciones y la cuestiones relativas al escaso interés turístico-residencial por la lejanía de la primera línea de costa, configuran un área urbana de reducido atractivo para la población en general que repercute a la postre en el abaratamiento del precio del suelo y de la vivienda y atrae a la población con menor poder adquisitivo. Sin lugar a dudas, los datos indican que se trata de la zona donde los problemas de exclusión social se dan de forma más acentuada y la población padece problemas de restricciones en el acceso a los servicios e ingresos necesarios para tener un nivel de vida aceptable. En principio, es un ejemplo muy evidente que confirma lo que otros autores ya señalaron (Wachinger et al., 2010) y que indica que, entre los factores de mayor influencia sobre la percepción y, por ende, sobre la vulnerabilidad social, estaría el de la experiencia personal de algún evento. En efecto, las zonas que mayor frecuencia de riadas son las más antiguas (localizadas en el centro de la ciudad) y las que cuentan ya con medidas de protección estruc- 


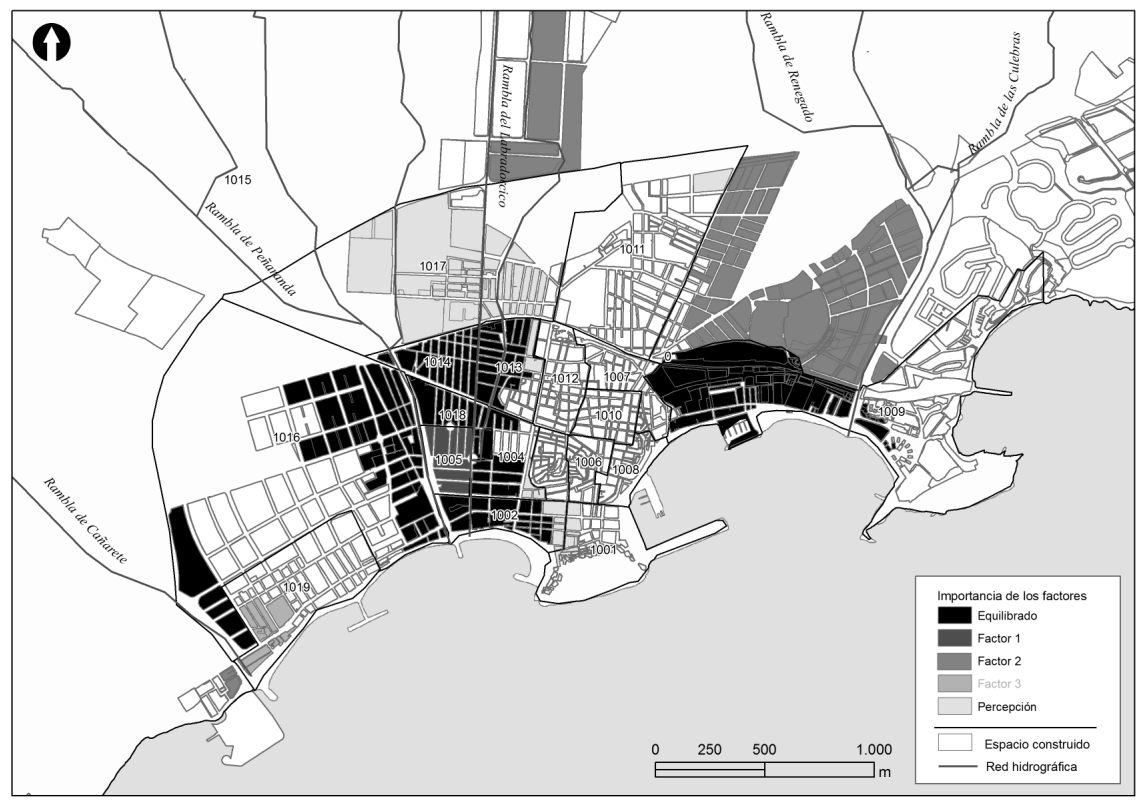

Figura 7. Importancia de los factores en el valor final de vulnerabilidad a partir de la clasificación.

Fuente: elaboración propia.

turales que refuerzan esta debilidad; por otro lado, está la población que vive en la zona más deprimida y donde las inundaciones son un fenómeno más reciente.

El resto de secciones presentan una vulnerabilidad y un predominio menores en cuanto al equilibrio en la significación de los factores en su valor final de vulnerabilidad; no obstante, el mapa permite distinguir algunas particularidades espaciales que acontecen en cuanto a lo destacado de la sección 1005 , 1015, y 1019, donde sobresalen los factores 1,2 y 3 , respectivamente.

\section{Conclusiones}

Primera. El método estadístico de análisis multivariante del ACP resulta válido para descubrir factores ocultos en grandes grupos de variables. Para el caso que nos ocupa, los resultados han confirmado la adecuación del mismo agrupando variables que tenían cierto grado de homogeneidad y conformando factores con sentido a las dimensiones de la vulnerabilidad. Sin embargo, cabe preguntarse ya no solo por la reducción, sino, más bien sobre la verdadera contribución de cada uno de ellos al concepto de vulnerabilidad social. La selección de los indicadores que describen con precisión la vulnerabilidad de una población ante un tipo de riesgo específico, e incluso, y más controvertido aún, la ponde- 
ración de dichos factores en el valor final es uno de los retos más importantes en este tipo de estudios (Cutter et al., 2000).

Segunda. Por otro lado, la cuestión de la percepción debe ser tratada con mucha más profundidad e incluida de forma necesaria en la construcción del marco conceptual que respalda este tipo de índices. En este primer trabajo, se han puesto de relevancia aspectos que podían pasar desapercibidos en una evaluación, en cuanto a que existen zonas que aún teniendo niveles bajos de vulnerabilidad, destacan por su escaso grado de percepción. Además, confirma lo que otros autores ya señalan, y es que la población con menos experiencia en catástrofes y más vulnerable, adolece de una percepción que le permita acometer medidas de protección para la mejora de esas condiciones. Aunque ya se sabe que la percepción contribuye en la vulnerabilidad social como el conjunto de una serie de acciones, resulta interesante evidenciar esa serie de debilidades o grietas que podrían acentuar ese proceso mediante la aplicación de este tipo de metodologías.

Tercera. Por último, los avances en la disposición de cartografía y fuentes estadísticas con alto grado de resolución espacial van permitiendo salvar las limitaciones de la escala a medida que aumenta y mejora la cantidad de información disponible a ese nivel de detalle. Como se ha puesto de relieve en el trabajo, la precisión catastral y el nivel de desagregación de los datos censales en secciones posibilitan realizar aproximaciones precisas muy útiles para averiguar la distribución de los procesos de vulnerabilidad en ámbitos muy concretos.

Cuarta. En cualquier caso, aunque es evidente que hay que depurar muchos aspectos para mejorar la precisión de los resultados, la cartografía y los índices de vulnerabilidad, considerados en el presente estudio, podrían convertirse en una herramienta factible y perfectamente aplicable que permite identificar y realizar un diagnóstico empírico sobre los distintos factores que pueden contribuir al aumento de la vulnerabilidad. En efecto, lo anterior facilita que se concentren y se dirijan con precisión los esfuerzos de forma sectorial a la hora de acometer las medidas necesarias para mitigar el factor de riesgo que afecta a una población o a un sector de la misma. La correcta integración de este tipo de cartografía dentro de los procesos de ordenación y planificación simplifica la tarea de los profesionales del territorio para sentar las bases de un modelo de desarrollo social y económico que, además de suavizar esas características negativas de marginación, restaura la situación de concierto y conformidad ante unos condicionantes naturales que están presentes en el medio y cuyo funcionamiento debe entenderse como parte de la naturaleza.

\section{Referencias bibliográficas}

Acosta-Michlik, L. (2005). Intervulnerability Assessment, Shifting Foci from Generic Indices to Adaptive Agents in Assessing Vulnerability to Global Environmental Change (A Pilot Project in the Philippines). Louvain-la-Neuve: University Catholic of Louvain (UCL), Department of Geography.

Adger, W. N. (2006). "Vulnerability». Global environmental change, 16 (3), 268-281. <http://dx.doi.org/10.1016/j.gloenvcha.2006.02.006> 
Alexander, M.; Faulkner, H.; Viavattene, C. y Priest, S. A. (2011). A GiS-based Flood Risk Assessment Tool: Supporting Flood Incident Management at the local scale. Research report. Flood Hazard Research Centre, Middlesex University

Balica, S. F.; Douben, N. y Wright, N. G. (2009). «Flood vulnerability indices at varying spatial scales». Water Science o Technology, 60 (10) 2571-2580. <http://dx.doi.org/10.2166/wst.2009.183>

Birkmann, J. (ed.) (2006). Measuring vulnerability to natural hazards: towards disaster resilient societies. Nueva York: United Nations University.

- (2007). «Risk and vulnerability indicators at different scales: applicability, usefulness and policy implications». Environmental Hazards, 7 (1), 20-31. <http://dx.doi.org/10.1016/j.envhaz.2007.04.002>

Blaikie, P.; Cannon, T.; Davis, I. y Wisner, B. (2014, 2a ed.). At risk: natural hazards, people's vulnerability and disasters. Londres: Routledge.

Burton, I. y Kates, R. W. (1964). «The Perception of Natural Hazards in Resource Management». Natural Resources Journal, vol. III, 3, 412-441.

Burton, I.; Kates, R. W. y White, G. F. (1968). «The human ecology of extreme geophysical events». Natural hazard research, 1-33.

Calvo García-Tornel, F. (2001). Sociedades y territorios en riesgo. Barcelona: Ediciones del Serbal (Colección La Estrella Polar, 31).

Calvo García-Tornel, F. y Granell Pérez, M. D. C. (2009). «Valoración social del riesgo por inundación en el litoral meridional de la Región de Murcia». Scripta Nova: Revista electrónica de geografía y ciencias sociales, 13, 281-309.

Camarasa Belmonte, A. M.; López García, M. J. y Soriano García, A. (2007). "Cartografía de vulnerabilidad frente a inundaciones en llanos mediterráneos: El caso de estudio del Barranco de Carraixet y Rambla del Poyo». Serie Geográfica, $14,75-91$.

Camarasa Belmonte, A. M. y López García, M. J. (2008). «Peligro, vulnerabilidad y riesgo de inundación en ramblas mediterráneas: los llanos de Carraixet y Poyo». Cuadernos de Geografía, 83, 1-26.

Camarasa-Belmonte, A. M., y Soriano-García, J. (2012). «Flood risk assessment and mapping in peri-urban Mediterranean environments using hydrogeomorphology. Application to ephemeral streams in the Valencia region (eastern Spain)». Landscape and Urban Planning, 104 (2), 189-200. <http://dx.doi.org/10.1016/j.landurbplan.2011.10.009>

Cardona, O. D. (2001). "La necesidad de repensar de manera holística los conceptos de vulnerabilidad y riesgo. Una crítica y una revisión necesaria para la gestión». En: Work-Conference on Vulnerability in Disaster Theory and Practice. Wegeningen, Disaster Studies of Wegeningen University and Research Center.

- (2005). System of Indicators for Disaster Risk Management, Program for Latin America and The Caribbean. Washington, D.C.: Main Technical Report, Inter-American Development Bank, Sustainable Development Department.

Chakraborty, J.; Tobin, G. A. y Montz, B. E. (2005). «Population Evacuation: Assessing Spatial Variability in Geophysical Risk and Social Vulnerability to Natural Hazards», Natural Hazards Review, (6) 1, 23-33. <http://dx.doi.org/10.1061/(ASCE)1527-6988(2005)6:1(23)>

Clark, G. E.; Moser, S. C.; Ratick, S. J.; et al. (1998). "Assessing the Vulnerability of Coastal Communities to Extreme Storms: The Case of Revere, MA, USA». Mitigation and Adaptation Strategies for Global Change, 3, 59-82. <http://dx.doi.org/10.1023/A:1009609710795> 
Coninx, I. y Bachus, K. (2007). «Integrating social vulnerability to floods in a climate change context». Retrieved April, 10, 1-26.

Cutter, S. L. (1996). «Vulnerability to Environmental Hazards». Progress in Human Geography, 20 (4), 529-539.

- (ed.) (2002). American Hazardscapes: The regionalization of hazards and disasters. Washington, D.C.: National Academies Press.

Cutter, S. L.; Mitchell, J. T. y Sсотт, M. S. (2000). «Revealing the vulnerability of people and places: a case study of Georgetown County, South Carolina». Annals of the Association of American Geographers, 90 (4), 713-737. <http://dx.doi.org/10.1111/0004-5608.00219>

Cutter, S. L.; Boruff, B. J. y Shirley, W. L. (2003). «Social vulnerability to environmental hazards». Social science quarterly, 84 (2), 242-261. <http://dx.doi.org/10.1111/1540-6237.8402002>

Cutter, S. L. y Finch, C. (2008). «Temporal and spatial changes in social vulnerability to natural hazards». Proceedings of the National Academy of Sciences, 105 (7), 2301-2306. <http://dx.doi.org/10.1073/pnas.0710375105>

Díez Herrero, A.; Garrote Revilla, J.; Baíllo Calvo, R.; et al. (2008). «Análisis del riesgo de inundación para planes autonómicos de protección civil: RICAM». En: Galindo Jiménez, I., Laín Huerta, L., Llorente Isidro, M. (eds.). El estudio y la gestión de los riesgos geológicos. Madrid: Instituto Geológico y Minero de España y Consorcio de Compensación de Seguros (MEH), 53-70.

Dwyer, A.; Zoppou, C.; Nielsen, O.; Day, S. y Roberts, S. (2004). Quantifying Social Vulnerability: A methodology for identifying those at risk to natural hazards. Geoscience Australia Record 2004/14. Australian Government, 92.

EIRD (2005). Marco de acción de Hyogo 2005-2015. Ginebra: Secretaría de la Estrategia Internacional de la ONU para la Reducción de los Desastres. <www.unisdr. org> [consulta 29 de julio de 2014]

Fekete, A. (2009). «Validation of a social vulnerability index in context to river-floods in Germany». Natural Hazards \& Earth System Sciences, 9 (2), 393-403. <http://dx.doi.org/10.5194/nhess-9-393-2009>

Fenete, A.; Damm, M. y Birkmann, J. (2010). «Scales as a challenge for vulnerability assessment». Natural Hazards, 55 (3), 729-747. <http://dx.doi.org/10.1007/s11069-009-9445-5>

Gall, M.; Borden, K. A. y Cutter, S. L. (2009). «When do losses count? Six fallacies of natural hazards loss data». Bulletin of the American Meteorological Society, 90 (6), 799-809. <http://dx.doi.org/10.1175/2008BAMS2721.1>

Gil-Guirado, S.; Pérez, A. y Barriendos, M. (2014). «Increasing vulnerability to flooding in the southern Spanish Mediterranean coast (1960-2013)». En: Hydrological extreme events in historic and prehistoric times, Bonn.

Hahn, H.; Villagrán De Leon, J. C.; Hidajat, R.; Schaef, T. y Bollin, C. (2003). Component III: Indicators and other disaster risk management instruments for communities and local governments. Washington, D.C.: Inter-American Development Bank.

Heinz Center for Science, Economics, and the Environment (2000). The Hidden Costs of Coastal Hazards: Implications for Risk Assessment and Mitigation. Washintong D. C.: Island Press.

Hewitt, K. (1997). Regions of Risk: A Geographical Introduction to Disasters. Essex: Longman. 
Holand, I. S. y Lujala, P. (2013). «Replicating and adapting an index of social vulnerability to a new context: a comparison study for Norway». The Professional Geographer, 65 (2), 312-328. <http://dx.doi.org/10.1080/00330124.2012.681509>

Instituto Nacional de Estadística (2001). Censo de población y viviendas de 2001. Secretaría de Estado de Economía y Apoyo a la Empresa. <http://www.ine.es> [consulta: 8 de enero de 2013].

Kates, R. W. (1962). Hazard and choice perception in flood plain management. Chicago: University of Chicago.

Kaztman, R. (2000). «Notas sobre la medición de la vulnerabilidad social». BID-Banco Mundial-CEPALIDEC, 5, 275-301.

Kleinosky, L. R.; Yarnal, B. y Fisher, A. (2007). "Vulnerability of Hampton Roads, Virginia to Storm-Surge Flooding and Sea-Level Rise». Natural Hazards, 40, 43-70. <http://dx.doi.org/10.1007/s11069-006-0004-z>

Lara, A.; Saurí, D.; Ribas, A. y Pavón, D. (2010). «Social perceptions of floods and flood management in a Mediterranean area (Costa Brava, Spain)». Natural Hazards and Earth System Sciences, 10 (10), 2081-2091.

Lara, A. (2012). Percepción social en la gestión del riesgo de inundación en un área mediterránea. Girona: Universitat de Girona. <http://hdl.handle.net/10803/98249> <http://hdl.handle.net/10256/7401>

Masgrau, L. R. y Palom, A. R. (2012). «Flood vulnerability and commercial activities: the case of the city of Girona, Spain». Disasters, 36 (4), 676-699. <http://dx.doi.org/10.1111/j.1467-7717.2012.01277.x>

Mileti, D. S. y O’Brien, P. W. (1992). «Warnings during disaster: Normalizing communicated risk». Social Problems, 39, 40-57. <http://dx.doi.org/10.2307/3096912>

Ministerio de Agricultura, Alimentación y Medio Ambiente. Sistema Nacional de Cartografía de Zonas Inundables. Servicio WMS. <http://www.magrama.gob. es/es/agua/temas/gestion-de-los-riesgos-de-inundacion/snczi/> [consulta: $16 \mathrm{de}$ marzo de 2014].

Ministerio de Hacienda y Administraciones Públicas. Dirección General del Catastro. WMS: <http://www.sedecatastro.gob.es> [consulta: 20 de marzo de 2014]

Morales Gil, A. (1968). "El riego con aguas de avenida en las laderas subáridas». Papeles del Departamento de Geografía, 1, 167-183.

Nakamura, T.; Hutton, C.; Kunbao, X. y Gavidia, J. (2001). Assessment of vulnerability to flood impacts and damages. UN. Centre for Human Settlements (Habitat). Disaster Management Programme.

NGo, E. B. (2001). "When disasters and age collide: Reviewing vulnerability of the elderly». Natural Hazards Review, 2 (2), 80-89. <http://dx.doi.org/10.1061/(ASCE) 1527-6988(2001)2:2(80)>

Olfert, A.; Greiving, S. y Batista, M. J. (2006). «Regional multi-risk review, hazard weighting and spatial planning response to risk - Results from European case studies. Natural and technological hazards and risks affecting the spatial development of European regions». Geological Survey of Finland, Special Paper 42, 125-151,

Olcina Cantos, J. (2004). «Riesgo de inundaciones y ordenación del territorio en la escala local El papel del planeamiento urbano municipal». Boletín de la AGE, 37, 49-84.

Parlamento Europeo. Directive 2007/60/EC on the assessment and management of flood risks in all available languages. <http://eur-lex.europa.eu/legal-content/EN/ TXT/?uri=CELEX\%3A32007L0060> [consulta: 9 de agosto de 2013]. 
Pérez Morales, A. (2008). «Aumento del riesgo de inundación por ocupación indebida de las áreas de convergencia de aguas en el Sur de la Región de Murcia». Scripta Nova. Revista electrónica de geografía y ciencias sociales, vol. XII, núm. 270 (27), Universidad de Barcelona.

Perles Roselló, M. J (2010). «Apuntes para la evaluación de la vulnerabilidad social frente al riesgo de inundación». Baetica. Estudios de Arte, Geografia e Historia, 32, 67-87.

Puente, S. (1999). "Social Vulnerability to Disaster in Mexico City». En: Mitchell, J. K. (ed.). Crucibles of Hazard: Mega-Cities and Disasters in Transition. Tokyo: United Nations University Press. 295-334

Rygel, L.; O’Sullivan, D. y Yarnal, B.(2006). «A Method for Constructing a Social Vulnerability Index». Mitigation and Adaptation Strategies for Global Change, 11, 741-764. <http://dx.doi.org/10.1007/s11027-006-0265-6>

Scheuer, S.; HaAse, D. y Meyer, V. (2011). «Exploring multicriteria flood vulnerability by integrating economic, social and ecological dimensions of flood risk and coping capacity: from a starting point view towards an end point view of vulnerability". Natural Hazards, 58 (2), 731-751. <http://dx.doi.org/10.1007/s11069-010-9666-7>

Scolobig, A.; De Marchi, B. y Borga, M. (2012). «The missing link between flood risk awareness and preparedness: findings from case studies in an Alpine Region». Natural Hazards, 63 (2), 499-520. <http://dx.doi.org/10.1007/s11069-012-0161-1>

Sebald, C. (2010). «Towards an integrated flood vulnerability index: A flood vulnerability assessment». Master of Science (MSc).

Smit, B., \& WANDEL, J. (2006). «Adaptation, adaptive capacity and vulnerability». Global environmental change, 16 (3), 282-292.

Tapsell, S. M.; Penning-Rowsell, E. C.; Tunstall, S. M. y Wilson, T. L. (2002). "Vulnerability to flooding: health and social dimensions». Philosophical transactions of the Royal Society of London / A, 360, 1511-1525. <http://dx.doi.org/10.1098/rsta.2002.1013>

Tapsell, S. M.; Tunstall, S. M.; Green, C. y Fernandez, A. (2005). Task 11 social indicator set. Tech. rep., FLOODsite report T11-07-01. <http://www.floodsite. net> [consulta: 16 de enero de 2013].

Tapsell, S.; McCarthy, S.; Faulkner, H. y Alexander, M. (2010). Social vulnerability to natural hazards CapHaz-Net's WP4-Report. Londres: Flood Hazard Research Centre (FHRC), Middlesex University.

Wachinger, G.; Renn, O.; Bianchizza, C. et al. (2010). Risk Perception and Natural Hazards. CapHaz-Net WP3. Institute for Communication and Cooperative Research, Stuttgart. <http://caphaz-net.org/outcomes-results/CapHaz-Net_WP3_ Risk-Perception2.pdf> [consulta: 15 de enero de 2013].

Weichselgartner, J. (2002). "About the capacity to be wounded: the need to link disaster mitigation and sustainable development». Extreme Naturereignisse-Folgen, Vorsorge, Werkzeuge. Bonn: DKKV, 150-158.

Wu, S. Y.; Yarnal, B. y Fisher, A. (2002). "Vulnerability of coastal communities to sea-level rise: a case study of Cape May county, New Jersey, USA». Climate Research, 22 (3), 255-270.

YARnal, B. (2007). "Vulnerability and all that jazz: Addressing vulnerability in New Orleans after Hurricane Katrina». Technoogy in Society, 29, 249-255.

<http://dx.doi.org/10.1016/j.techsoc.2007.01.011> 
Zahran, S.; Brody, S. D.; Peacock, W. G.; Vedlitz, A. y Grover, H. (2008). "Social vulnerability and the natural and built environment: a model of flood casualties in Texas». Disasters, 32 (4), 537-560.

<http://dx.doi.org/10.1111/j.1467-7717.2008.01054.x>

\section{Apéndice}

Variables que han intervenido en la construcción del índice por secciones afectadas según el periodo de retorno de 500 años.

\begin{tabular}{|c|c|c|c|c|c|c|c|c|c|c|c|c|c|c|c|c|}
\hline \multirow{2}{*}{$\begin{array}{l}\text { Secciones } \\
\text { afectadas }\end{array}$} & \multicolumn{2}{|c|}{ Población } & \multicolumn{4}{|c|}{ Edad } & \multirow{2}{*}{\multicolumn{2}{|c|}{$\frac{\text { Feminidad }}{\text { FEM }}$}} & \multicolumn{6}{|c|}{ Hogares } & \multicolumn{2}{|c|}{ Discapacidad } \\
\hline & Pob & $\%$ & $\mathrm{Pob}<4$ & $\% \quad \mathrm{P}$ & Pob $>65$ & $5 \quad \%$ & & & \multicolumn{2}{|c|}{$\mathrm{n}^{0}$ hogares } & $\mathrm{HOG}>64$ & \multicolumn{2}{|r|}{ HOGMON } & $\%$ & DISCA & $\%$ \\
\hline 1002 & 1273 & 4,6 & 74 & 5,8 & 210 & 16 & & 113 & 437 & & 88 & 22,4 & 9 & 2,1 & 112 & 0,4 \\
\hline 1004 & 2127 & 7,7 & 115 & 5,4 & 328 & 15 , & & 108 & 679 & & & 16,1 & 9 & 1,3 & 187 & 0,7 \\
\hline 1005 & 1214 & 4,4 & 79 & 6,5 & 183 & 15 , & & 101 & 372 & & 5 & 12,1 & 6 & 1,6 & 107 & 0,4 \\
\hline 1009 & 1595 & 5,7 & 60 & 3,8 & 302 & 18 , & & 96 & 566 & & & 23,5 & 8 & 1,4 & 140 & 0,5 \\
\hline 1011 & 2906 & 10,5 & 210 & 7,2 & 342 & 11 & & 100 & 801 & & 99 & 8,6 & 12 & 1,5 & 256 & 0,9 \\
\hline 1013 & 2137 & 7,7 & 111 & 5,2 & 263 & 12 & & 103 & 630 & & 77 & 9,0 & 7 & 1,1 & 188 & 0,7 \\
\hline 1014 & 1150 & 4,1 & 51 & 4,4 & 166 & 14 & & 94 & 343 & & 3 & 12,5 & 5 & 1,5 & 101 & 0,4 \\
\hline 1015 & 1136 & 4,1 & 31 & 2,7 & 226 & 19 , & & 92 & 459 & & 7 & 10,2 & 6 & 1,3 & 100 & 0,4 \\
\hline 1016 & 1932 & 7,0 & 131 & 6,8 & 141 & 7, & & 96 & 592 & & 55 & 9,3 & 9 & 1,5 & 170 & 0,6 \\
\hline 1017 & 1228 & 4,4 & 99 & 8,1 & 78 & 6 , & & 92 & 309 & & 0 & 12,9 & 9 & 2,9 & 108 & 0,4 \\
\hline 1018 & 1617 & 5,8 & 1227 & 7,5 & 118 & 7 , & & 101 & 463 & & 2 & 9,1 & 10 & 2,2 & 142 & 0,5 \\
\hline 1019 & 1502 & 5,4 & 115 & 7,7 & 143 & 9 , & & 103 & 487 & & 34 & 17,2 & 11 & 2,3 & 132 & 0,5 \\
\hline 1018 & 57 & 3,5 & 10 & 0,6 & 98 & 6 , & & 50 & 3,09 & & & 6,8 & 421 & 26,0 & 47 & 2,9 \\
\hline \multirow[t]{2}{*}{1019} & 28 & 1,9 & 1 & 0,1 & 81 & 5, & & 37 & 2,46 & & 31 & 12,1 & 210 & 14,0 & 19 & 1,3 \\
\hline & \multicolumn{5}{|c|}{ Extranjeros } & \multicolumn{9}{|c|}{ 0cupación } & \multicolumn{2}{|c|}{ Estudios } \\
\hline $\begin{array}{l}\text { Secciones } \\
\text { afectadas }\end{array}$ & Ext & $\%$ & Extinf & $\%$ & $\%$ & Paro & $\%$ & Paro juv. & $\%$ & Ocu. ev. & $\%$ & & U. NC & $\%$ & Analf. & $\%$ \\
\hline 1002 & 90 & & 10 & 0,8 & 8 & 70 & 5,5 & 25 & 1,96 & 189 & 14,8 & & 179 & 14,1 & 23 & 1,8 \\
\hline 1004 & 70 & & 10 & 0,5 &, 5 & 78 & 3,7 & 47 & 2,21 & 258 & 12,1 & & 306 & 14,4 & 43 & 2,0 \\
\hline 1005 & 57 & & 4,7 & 0,7 & 1,7 & 83 & 6,8 & 37 & 3,05 & 177 & 14,6 & & 205 & 16,9 & 61 & 5,0 \\
\hline 1009 & 61 & & 3,8 & 0,4 &, 4 & 97 & 6,1 & 37 & 2,32 & 192 & 12,0 & & 172 & 10,8 & 16 & 1,0 \\
\hline 1011 & 38 & & 1,3 & 0,2 &, 2 & 107 & 3,7 & 57 & 1,96 & 715 & 24,6 & & 599 & 20,6 & 138 & 4,7 \\
\hline 1013 & 66 & 6 & 3,1 & 0,4 &, 4 & 110 & 5,1 & 61 & 2,85 & 196 & 9,2 & & 506 & 23,7 & 88 & 4,1 \\
\hline 1014 & 10 & & 0,9 & 0,1 &, 1 & 47 & 4,1 & 32 & 2,78 & 87 & 7,6 & & 211 & 18,3 & 14 & 1,2 \\
\hline 1015 & 247 & $7 \quad 21$, & 1,7 & 0,8 &, 8 & 42 & 3,7 & 16 & 1,41 & 96 & 8,5 & & 118 & 10,4 & 161 & 14,2 \\
\hline 1016 & 3 & & 1,6 & 0,3 & ,3 & 63 & 3,3 & 27 & 1,40 & 203 & 10,5 & & 199 & 10,3 & 29 & 1,5 \\
\hline 1017 & 4 & 3, & 14 & 1,1 & ,1 & 76 & 6,2 & 38 & 3,09 & 121 & 9,9 & & 376 & 30,6 & 117 & 9,5 \\
\hline 1018 & 57 & & 10 & 0,6 & ,6 & 98 & 6,1 & 50 & 3,09 & 110 & 6,8 & & 421 & 26,0 & 47 & 2,9 \\
\hline 1019 & 28 & & 1,9 & 0,1 &, 1 & 81 & 5,4 & 37 & 2,46 & 181 & 12,1 & & 210 & 14,0 & 19 & 1,3 \\
\hline
\end{tabular}

